\title{
Effect of pulsed heat treatment on structural and thermomechanical properties of rapidly quenched TiNiCu alloys
}

\author{
A. Shelyakov ${ }^{1 \mathrm{a}}$, N. Sitnikov ${ }^{1}$, N. Resnina ${ }^{2}$, A. Glezer ${ }^{3}$, and A. Korneev ${ }^{1}$ \\ ${ }^{1}$ Moscow Engineering Physics Institute (State University), Kashirskoe shosse 31, \\ 115409, Moscow, Russia \\ ${ }^{2}$ Research Institute of Mathematics and Mechanics, Mathematics and Mechanics Faculty, \\ Saint-Petersburg State University, Universitetsky pr. 28, 198504, Saint-Petersburg, Russia \\ ${ }^{3}$ G.V.Kurdyumov Institute of Physical Metallurgy, I.P.Bardin Science Center of Ferrous Metallurgy, \\ Vtoraya Baumanskaya ul. 9/23, 105005, Moscow, Russia
}

\begin{abstract}
The present work is concerned with the investigation of shape memory $\mathrm{Ti}_{50} \mathrm{Ni}_{25} \mathrm{Cu}_{25}$ (at.\%) alloy fabricated by melt spinning technique. The parameters of technology were optimized to produce the alloy in amorphous state. Dynamic crystallization of the alloy was performed by single electric pulse with duration from $2 \mathrm{~ms}$ to $100 \mathrm{~ms}$. TEM and DSC investigations shown that the decrease of the pulse duration down to 2 ms resulted in significant refinement of the alloy structure with formation of nanosized martensitic plates $(20-60 \mathrm{~nm})$, and to decrease in critical temperatures of martensite transformations. The shape memory behavior of the studied alloy was characterized by strain-temperature curves obtained by thermal cycling the specimens through the transformation range under constant stress. It was found that the nanostructurization of the alloy led to some increase of completely recovery strain.
\end{abstract}

\section{Introduction}

In recent years, shape memory alloys (SMAs) have impressively entered in such application fields like power, instrument and mechanical engineering, aerospace technology, and robotics [1-4]. New arising engineering needs require small, cheap and fast responding devices based on such alloys. This seriously motivates the necessity to develop new thin ultradispersive SMA materials.

The characteristic property of rapidly quenched alloys of the quasi-binary TiNi-TiCu system with the high $\mathrm{Cu}$ contents, produced by melt-spinning and planar flow casting techniques, is that at high cooling rates these alloys can be obtained in amorphous state [5]. After a heat treatment the alloys exhibit pronounced shape memory effect (SME) which substantially depends on structural parameters of the alloys. This paper presents investigation of the influence of crystallization regimes of amorphous alloys on their microstructure and thermomechanical properties.

\section{Experimental}

The material for this study was manufactured by a single-roller melt-spinning technique from presynthesized $\mathrm{Ti}_{50} \mathrm{Ni}_{25} \mathrm{Cu}_{25}$ (at.\%) alloys. High purity nickel, titanium and cooper were melted six times in an argon arc furnace. The obtained ingots were re-molten in quartz crucibles under a purified atmosphere of inert gas and ejected onto the surface of a fast rotating copper wheel at cooling rates around $10^{6} \mathrm{~K} / \mathrm{s}$. Thus, the rapidly solidified $\mathrm{Ti}_{50} \mathrm{Ni}_{25} \mathrm{Cu}_{25}$ alloys were prepared in the form of continuous (10-30 meters long) ribbons with a thickness in the range $30-50 \mu \mathrm{m}$ and a width around $1.5 \mathrm{~mm}$.

Alloy samples were dynamically crystallized via single electric pulse application. In accordance with performed calculations, pulse amplitude and duration were chosen to provide heat energy release that is required for heating the sample up to crystallization temperature. A number of the samples with time of crystallization in the range from 1 to $100 \mathrm{~ms}$ were prepared. For comparison some alloy samples were crystallized by standard isothermal heat treatment in a furnace at $500^{\circ} \mathrm{C}$ in $240 \mathrm{~s}$.

aemail: alex-shel@mail.ru

This is an Open Access article distributed under the terms of the Creative Commons Attribution-Noncommercial License (http://creativecommons.org/licenses/by-nc/3.0/), which permits unrestricted use, distribution, and reproduction in any noncommercial medium, provided the original work is properly cited. 
The microstructure observations were performed using a TESLA BS-540 transmission electron microscope. The phase transformation intervals were determined by plotting electric resistance versus temperature curves and by differential scanning calorimetry (DSC) using a Mettler Toledo $822^{\mathrm{e}}$ instrument at heating/cooling rate of $10^{\circ} \mathrm{C} / \mathrm{min}$. Shape memory behavior of the studied melt spun $\mathrm{TiNiCu}$ alloys was characterized by straintemperature curves obtained by thermal cycling of samples through the transformation range under constant stress.

\section{Results}

The melt-spinning process for studied alloys produced mainly amorphous state of the ribbons after solidification as revealed by TEM investigations (Fig.1a). Isothermal crystallization of the alloys during heating in furnace up to $500^{\circ} \mathrm{C}$ results in formation of a metastable B2-phase structure. Cooling down to room temperature, the martensitic transformation induces on orthorhombic martensite B19 which TEM images and diffraction pattern are presented in Fig.1b. The average grain size is $300 \div 500 \mathrm{~nm}$, in good agreement with results obtained in [6].
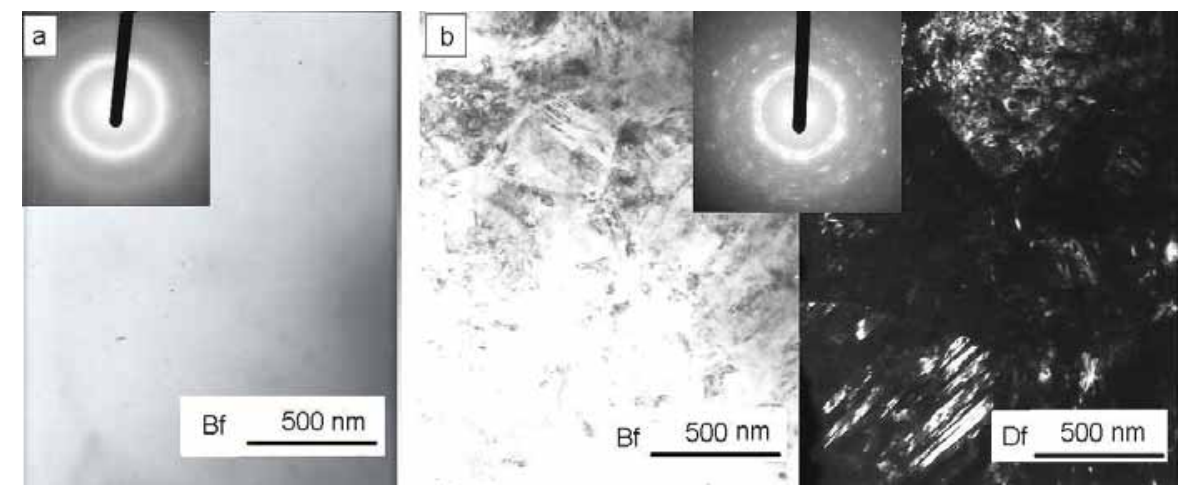

Fig. 1. TEM micrographs and selected-area electron diffraction patterns of a $\mathrm{Ti}_{50} \mathrm{Ni}_{25} \mathrm{Cu}_{25}$ (at.\%) alloy at an initial asquenched state (a) and after isothermal crystallization (b)

In dynamically crystallized samples, even for crystallization times of $100 \mathrm{~ms}$, it was found a modification of the crystallization mechanism characterized by the formation of colonies of nanosized crystals. Decreasing the crystallization time down to $2 \mathrm{~ms}$, fine $20 \div 60 \mathrm{~nm}$ thick martensite plates appear. At the same time phase structure of the alloy is not changed and like in isothermically crystallized samples, the B2 $\rightarrow$ B19 martensitic transformation undergoes.
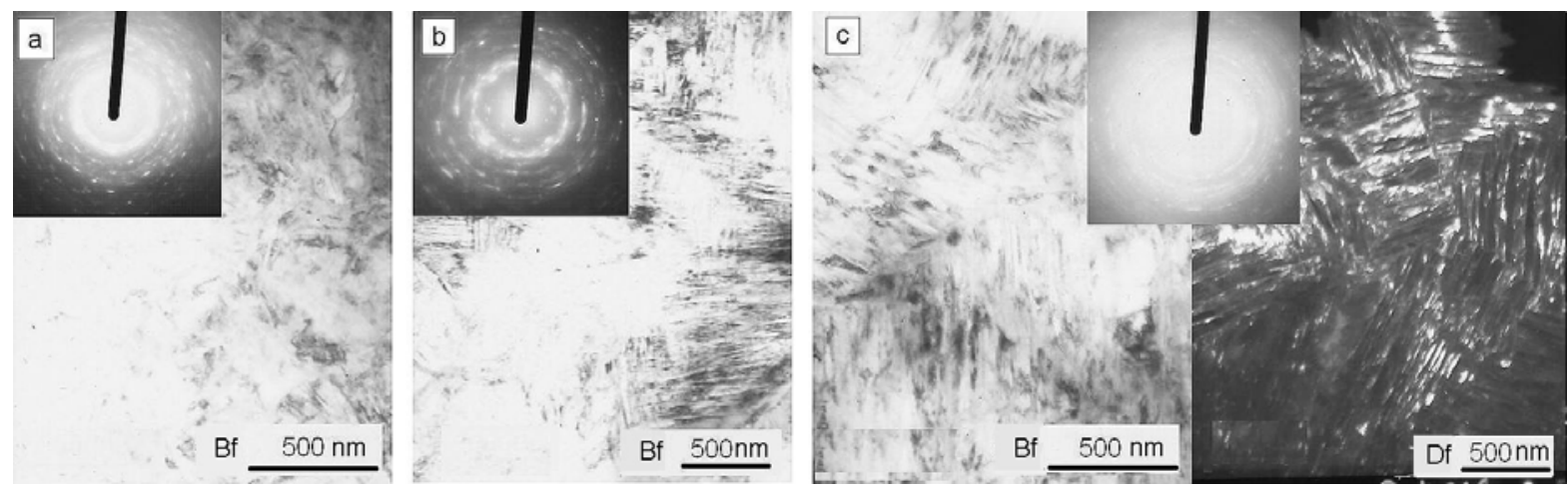

Fig. 2. TEM micrographs and selected-area electron diffraction patterns of rapidly quenched $\mathrm{Ti}_{50} \mathrm{Ni}_{25} \mathrm{Cu}_{25}$ (at.\%) alloy after dynamic crystallization at different times: $100 \mathrm{~ms}(\mathrm{a}), 10 \mathrm{~ms}$ (b) and $2 \mathrm{~ms}$ (c)

The investigation of temperature intervals of crystallization and martensitic transformation via the DSC method allows to estimate both the critical temperatures of phase transformations and the respective transformation enthalpy, characterizing the crystallization order and SME potential. Heating the as-quenched sample up to $600^{\circ} \mathrm{C}$ at $20^{\circ} \mathrm{C} / \mathrm{min}$ rate revealed no phase transformation peaks below $400^{\circ} \mathrm{C}$, while at $427^{\circ} \mathrm{C}$ a devitrification took place with the subsequent crystallization. The crystallization was comprised in the 457$464^{\circ} \mathrm{C}$ temperature interval with the peak at $461^{\circ} \mathrm{C}$, and the crystallization enthalpy constituted $34.9 \mathrm{~J} / \mathrm{g}$. 
For the samples of $\mathrm{Ti}_{50} \mathrm{Ni}_{25} \mathrm{Cu}_{25}$ alloy crystallized at different rates, heating and cooling cycles in the range from $20^{\circ} \mathrm{C}$ to $100^{\circ} \mathrm{C}$ were performed at $10^{\circ} \mathrm{C} / \mathrm{min}$ rates (Fig.3). It can be seen from the DSC curves that no phase transformation were present in as-quenched samples. The alloy crystallization, on the other hand, induces characteristic heat absorption-emission peaks on the DSC curves in the $55-80^{\circ} \mathrm{C}$ temperature interval.
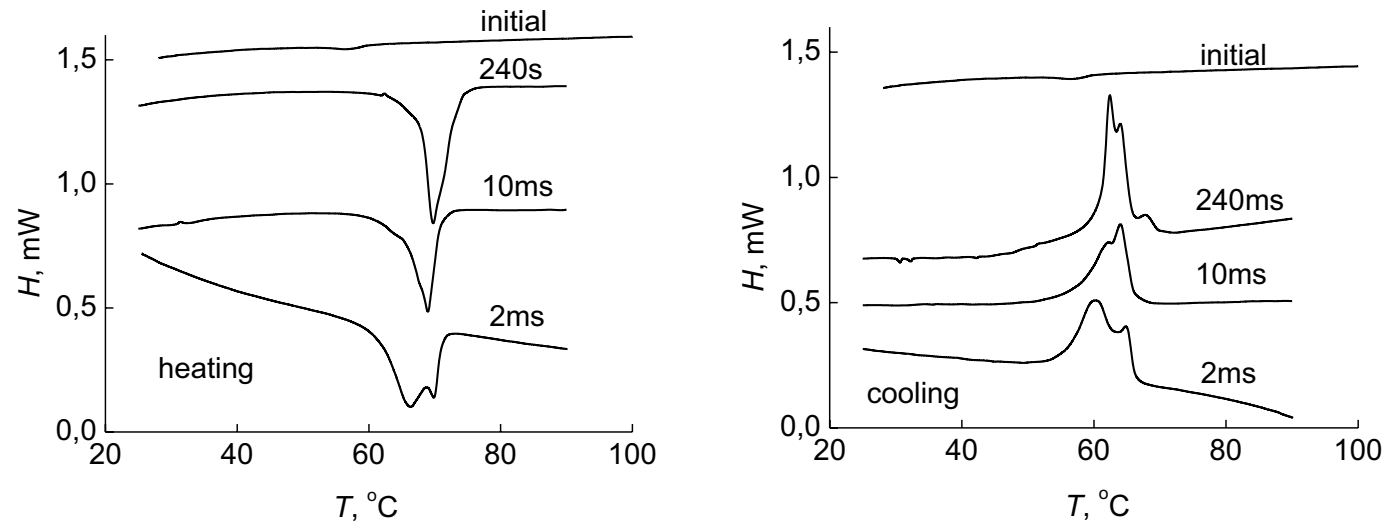

Fig. 3. DSC curves of $\mathrm{Ti}_{50} \mathrm{Ni}_{25} \mathrm{Cu}_{25}$ (at.\%) melt-spun ribbons crystallized at different times

It was established that increasing the crystallization rate leads to a small decrease of the critical temperatures and hysteresis of martensitic transformations by $2-4^{\circ} \mathrm{C}$. It has to be noted that for short crystallization times a twostage behavior of the transformation process was found. This indicates perhaps that the alloy is characterized by a bi-modal distribution of the grain sizes, i.e. a simultaneous presence of nano- and larger sized crystals is realized as observed by TEM investigation too.

The investigation of the temperature intervals via electrical resistance measurements showed that decreasing the crystallization times lowers the critical temperature. This is in a good agreement with DSC data. In order to investigate the thermo-mechanical properties of the alloy the samples were thermally cycled under a constant load. The dependence of the bending strain of the sample is indicated in the Fig. 4. The analysis of the reversible transformation strain provides the information of the shape recovery properties of the sample. It can be seen from the obtained curves that for dynamically crystallized ultradispersive samples, the transformation was at least not worse than in the isothermally crystallized sample, while in some cases the transformation strain exceeded the latter by $10 \%$. In addition, it was found that decreasing the crystallization time leads to a lower shape recovery temperature. It has to be mentioned that higher shape recovery temperatures are achieved for increased constant stress (Fig. 5).

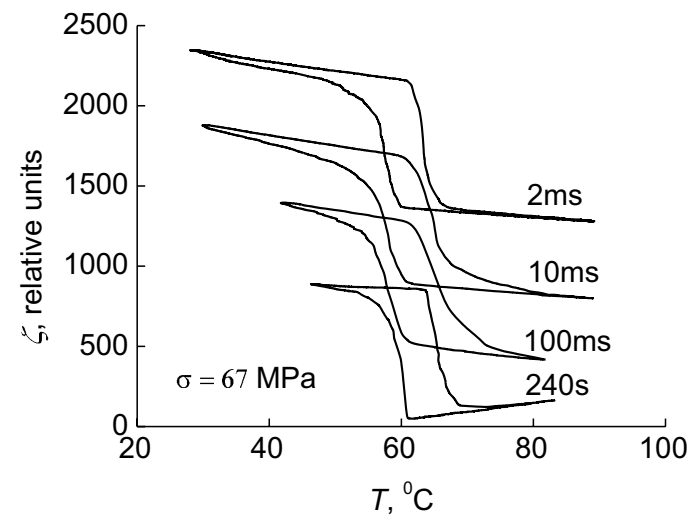

Fig. 4. Strain vs. temperature curves under constant stress of $\mathrm{Ti}_{50} \mathrm{Ni}_{25} \mathrm{Cu}_{25}$ melt-spun ribbons

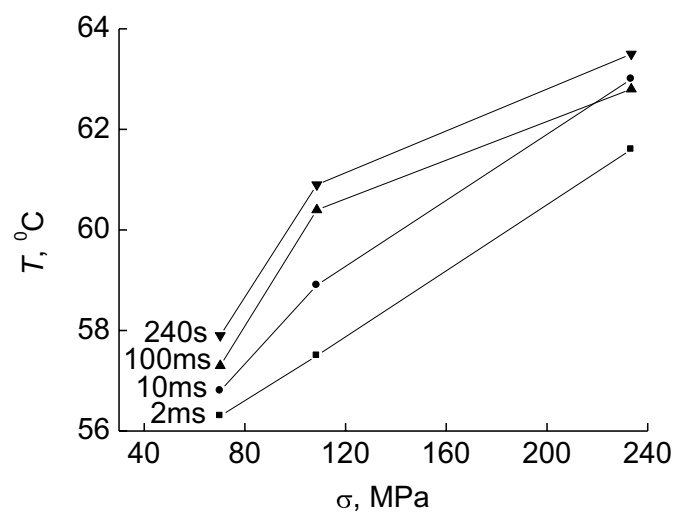

Fig. 5. Effect of constant stress on start temperature $M_{S}$ of martensitic transformation in RQ $\mathrm{Ti}_{50} \mathrm{Ni}_{25} \mathrm{Cu}_{25}$ alloy 


\section{Conclusions}

TEM and DSC investigations have shown that the crystallization of the amorphous $\mathrm{Ti}_{50} \mathrm{Ni}_{25} \mathrm{Cu}_{25}$ alloy leads to the formation of the B2 phase for all the heat treatment regimes, while at cooling the B2-B19 phase transition occurs. Reducing the time of the dynamic crystallization down to $2 \mathrm{~ms}$ results in a considerable refinement of the alloy structure. This is accompanied by development of nanosized martensitic plates (20-60 nm) and by a small decrease of the critical temperature of the phase transformation by $2-4^{\circ} \mathrm{C}$. The thermo-mechanical measurements confirmed that the nanostructurization of the alloy can enhance the reversible transformation strain by about $10 \%$.

\section{References}

[1] F.Yongqing, D.Hejun, H.Weimin et al., Sensors and Actuators A112, 395 (2004)

[2] J.Van Humbeeck, Materials Science and Engineering A273-275, 134 (1999)

[3] K.Otsuka, X.Ren, Intermetallics 7, 511 (1999)

[4] A.V.Shelyakov, V.A.Antonov, Yu.A.Bykovsky et al., "Optical devices based on shape memory effect for signal processing", Int. Conf. on Shape Memory and Superelastic Technologies (SMST-94), Pacific Grove, CA (USA), 7-10 March 1994, edited by A.R.Pelton, D.Hodgson and T.Duerig (MIAS, Monterey, CA, 1995), p. 335

[5] A.V.Shelyakov, S.G.Larin, V.P.Ivanov et al., J. Phys. IV France 11 Pr8-547 (2001)

[6] N.M.Matveeva , V.G.Pushin, A.V.Shelyakov et al., The Physics of Metals and Metallography 83, 626 (1997)

[7] N.Resnina, S.Belyaev, A.Shelyakov, The European Physical Journal - Special Topics 158, 21 (2008) 